\section{Commentary: A call-to-arms: Shifting culture in cardiothoracic surgery}

\author{
Brian Mitzman, MD
}

Are "health" and "wellness" synonyms or actually distinct concepts? The Oxford Dictionary describes health as "the state of being free from illness or injury." Wellness goes one step further. It includes happiness, and in some descriptions, even hedonism. It is achieved by "a combination of emotional, environmental, mental, physical, social, and spiritual health." In this expert opinion, Fajardo and colleagues ${ }^{1}$ explore wellness in the field of cardiothoracic surgery, and specifically why it has been a challenge to achieve wellness among our trainees. This is a call-toaction: A plea to our community to take the first step in developing guidelines and spread culture change in our field.

Fajardo and colleagues start this piece off with some sobering statistics - a suicide rate among physicians that is 1.41 and 2.27 times greater for men and women than the general population, and $51.5 \%$ of trainees who meet criteria for burnout. Even those who only care about the bottom line can't ignore these statistics-physician burnout in the United States costs $\$ 4.6$ billion dollars annually. Why aren't hour restrictions and more of a direct focus on trainee education enough? What makes cardiothoracic surgery trainees more prone to burnout? Let's explore.

The 2003 Accreditation Council for Graduate Medical Education 80-hour work week restrictions were supposed to improve resident wellness. Studies have shown that this goal likely was not achieved. ${ }^{2}$ Look at the components of wellness again. Did it improve environmental and physical health? Probably. It's hard to argue that physically a 16-hour shift isn't better than a $30+$ hour shift. What about the other

From the Department of Cardiothoracic Surgery, NYU Langone Health, New York, NY.

Disclosures: Author has nothing to disclose with regard to commercial support.

Received for publication March 29, 2020; revisions received March 29, 2020; accepted for publication March 30, 2020; available ahead of print April 10, 2020.

Address for reprints: Brian Mitzman, MD, Department of Cardiothoracic Surgery, NYU-Winthrop Hospital, 259 1st St, Mineola, NY 11501 (E-mail: Brian.

Mitzman@nyulangone.org).

J Thorac Cardiovasc Surg 2021;161:338-9

$0022-5223 / \$ 36.00$

Copyright (c) 2020 by The American Association for Thoracic Surgery

https://doi.org/10.1016/j.jtcvs.2020.03.129

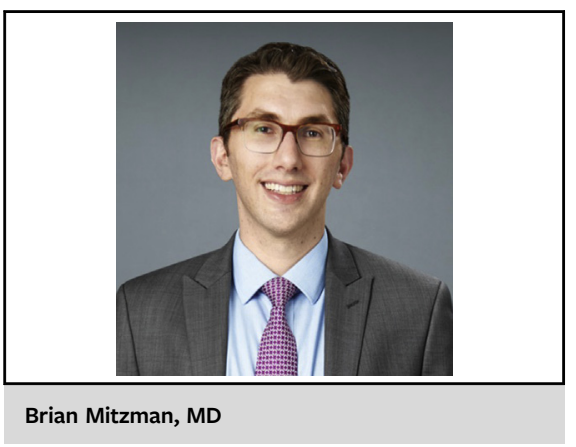

CENTRAL MESSAGE

Wellness is poorly defined and often misinterpreted as physical health. We must first understand wellness as a concept and then develop an action plan to prevent burnout of our trainees.

components, though-mental, emotional, and social? These hour restrictions often decreased formal education, decreased engagement with the team and supervisor, and increased resident anxiety that training would not be sufficient. ${ }^{3}$ This does not mean that the hour restrictions are unhelpful; it just means we need to contemplate wellness more deeply and do more.

What makes cardiothoracic surgery training specifically so challenging? Besides the obvious, Fajardo and colleagues appropriately note that the length of training compared with other fields is unique. Due to the competitiveness of obtaining a traditional fellowship slot, most residents complete 2 years of research in addition to 5 years of general surgery. This is followed by a 2- to 3-year cardiothoracic surgery fellowship, and then potentially an additional super-fellowship year. For those not counting, that's up to 11 years of training, putting many in our field at 37 years old before having any true financial stability or ability to focus on non-medical endeavors. Learning how to balance family life and childcare during training is commonplace, if not universal, as compared with fields with shorter training periods, such as internal medicine or emergency medicine.

Without society buy-in and institutional support, our trainees are guaranteed some degree of burnout. ${ }^{4}$ It is our responsibility to be proactive and develop a wellness strategy. As Fajardo and colleagues conclude, this is an investment in the future of our field needing immediate action. 


\section{References}

1. Fajardo R, Vaporciyan A, Starnes S, Erkmen CP. Cardiothoracic surgery wellness: now and the formidable road ahead. J Thorac Cardiovasc Surg. 2021;161:333-7.

2. Gelfand DV, Podnos YD, Carmichael JC, Saltzman DJ, Wilson SE, Williams RA. Effect of the 80-hour workweek on resident burnout. Arch Surg. 2004;139:933-40.
3. Ahmed N, Devitt KS, Keshet I, Spicer J, Imrie K, Feldman L, et al. A system atic review of the effects of resident duty hour restrictions in surgery: impact on resident wellness, training, and patient outcomes. Ann Surg. 2013;259: 1041-53.

4. Wallace JE, Lemaire JB, Ghali WA. Physician wellness: a missing quality indicator. Lancet. 2009;374:1714-21.
See Article page 333

\section{Commentary: Burning bright without burning out: Protecting the spirit of cardiothoracic surgery}

\author{
Jason J. Han, MD, John J. Kelly, MD, and \\ Rita Karianna Milewski, MD, PhD
}

Cardiothoracic surgery is a demanding specialty for which career success has long relied on resilience, ingenuity, and a tireless work ethic. The field will continue to attract individuals with these characteristics and thrive, but during the past several decades we have collectively grown more aware of the importance of promoting wellness and mitigating burnout among providers. Recent studies have demonstrated that burnout is associated with increased rates of provider depression, suicidal ideation, and attrition, as well as overall worse patient satisfaction and outcomes. ${ }^{1-5}$ These symptoms are far more prevalent among health care workers than in the general population. Most studies estimate that a majority of providers experience some degree of burnout during training or throughout their career, which points to a systemic problem in medicine. ${ }^{4,5}$ The coronavirus disease 2019 pandemic already has and will undoubtedly continue to exacerbate these associations

\footnotetext{
From the Division of Cardiovascular Surgery, Department of Surgery, Hospital of the University of Pennsylvania, Philadelphia, Pa.

Disclosures: Dr Milewski is a consultant for Destiny Pharma. All other authors reported no conflicts of interest.

The Journal policy requires editors and reviewers to disclose conflicts of interest and to decline handling or reviewing manuscripts for which they may have a conflict of interest. The editors and reviewers of this article have no conflicts of interest.

Received for publication April 17, 2020; accepted for publication April 17, 2020; available ahead of print May 1, 2020.

Address for reprints: Rita Karianna Milewski, MD, PhD, Division of Cardiovascular Surgery, Department of Surgery, Hospital of the University of Pennsylvania, 3400 Spruce St, 6 Silverstein Pavilion, Philadelphia, PA 19104 (E-mail: Rita.Milewski@ pennmedicine.upenn.edu).

J Thorac Cardiovasc Surg 2021;161:339-40

$0022-5223 / \$ 36.00$

Copyright (c) 2020 by The American Association for Thoracic Surgery

https://doi.org/10.1016/j.jtcvs.2020.04.081
}

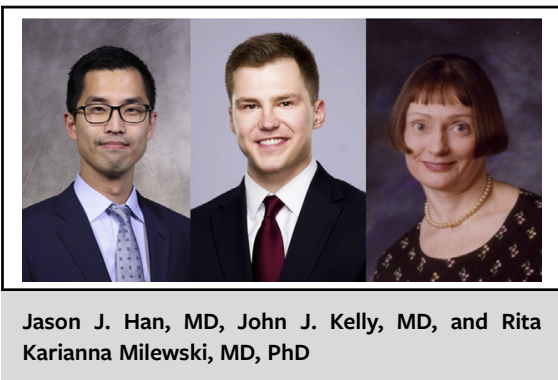

CENTRAL MESSAGE

Reducing burnout and promoting wellness among surgeons and trainees in cardiothoracic surgery should be a systemwide priority and requires a centralized solution.

at all levels of the health care system. This requires our attention.

It is at this necessary junction that Fajardo and colleagues $^{6}$ share their insights. Incorporating the wisdom of past and present leaders from the Thoracic Surgery Director's Association, the authors call for a top-down paradigm shift consisting of more robust, uniform definitions; establishment of institutional support and responsibility; increased research; and improved implementation of existing resources across programs. The strength of the article is in its bold and overarching vision that recognizes a need for a centralized approach toward creating a multi-institutional committee, mission statement, educational curriculum, and intervention plan. These suggestions can surely be the building blocks for an infrastructure surrounding wellness in our specialty. Yet, the other half of the 